\title{
Objects and attributes in divided attention: Surface and boundary systems
}

\author{
JOHN DUNCAN and IAN NIMMO-SMITH \\ MRC Applied Psychology Unit, Cambridge, England
}

\begin{abstract}
A series of experiments investigated concurrent discriminations of surface and nonsurface attributes, including color, brightness, texture, length, location, and motion. In all cases but one, results matched those previously reported: Interference occurred when two discriminations concerned different objects, but not when they concerned the same one. In the two-object case, interference was the same whether discriminations were similar (e.g., two surface discriminations) or different (e.g., one surface, one boundary). Such results support a model of visual attention in which separate visual subsystems are coordinated, converging to work on surface and boundary properties of the same selected object. A partial exception is color: For reasons that are unclear, color escapes twoobject interference except from other, concurrent surface discriminations.
\end{abstract}

Over the past $10-15$ years, there has been increasing emphasis on the modularity of the visual system and, in particular, the specialization of subsystems for analysis of different visual attributes, such as location, motion, color, and shape. Especially influential has been neurophysiological evidence for multiple extrastriate areas dealing at least in part with different kinds of visual information (Desimone \& Ungerleider, 1989; Zeki, 1978).

Following on from the early suggestions of Lappin (1967), Treisman (1969), and Allport (1971), a recent series of studies has investigated the implications of visual modularity for divided attention (Duncan, 1993a, 1993b). When two visual discriminations must be made concurrently, does it matter whether they involve the same or different attributes? In the different-attribute case, can distinct visual subsystems work independently and in parallel, reducing or eliminating the interference normally seen in divided attention?

In these experiments, subjects make two concurrent visual discriminations on the basis of a brief visual display. Each discrimination is a two-alternative forced choice, made without time pressure. Results to date have been straightforward. When concurrent discriminations concern different objects, there is interference between them. This interference is exactly the same whether they concern different attributes (e.g., identifying the orientation of one object and the location of another) or the same attribute. So far, there is no evidence for parallel, independent function of distinct subsystems.

Results are very different when discriminations concern different attributes of the same object (see also Duncan, 1984; Kahneman \& Henik, 1977). In this case inter-

This research was supported by a grant from the Human Frontier Science Program. Correspondence should be addressed to J. Duncan, MRC Applied Psychology Unit, 15 Chaucer Rd., Cambridge CB2 2EF, England (e-mail: john.duncan@mrc-apu.cam.ac.uk). ference is eliminated. The results support what Duncan (1984) has called object-based models of visual attention. "Attending" to an object is a state in which multiple visual subsystems converge to work on its different properties, making them concurrently available for report and control of behavior. Neurophysiological, neuropsychological, and behavioral evidence in support of this view has been summarized by Duncan (1996); the general idea, however, is much older (e.g., Broadbent, 1958; Neisser, 1967; Treisman \& Souther, 1985). Objects compete to enter the "attended" state, giving rise to interference when concurrent discriminations concern different objects ("divided attention"). This competition might be modeled as division of a fixed pool of activation or processing capacity (Bundsen, 1990; Rumelhart, 1970). According to this model, the key consideration in divided attention is simply the number of relevant objects in a display, not the nature or similarity of discriminations to be made (Duncan, 1993b).

The present experiments sought to extend previous findings by considering concurrent discriminations of surfaces and boundaries. A general distinction between surface and boundary systems has often been drawn (e.g., Grossberg, 1987; Livingstone \& Hubel, 1987). Boundary systems indicate divisions between one region (e.g., one object) and another; they could be used as the basis for such discriminations as shape, size, orientation, or object location. Surface systems fill in the regions thus demarcated with additional properties: color, brightness, texture, and so on. The surface-boundary distinction has an especially strong physiological motivation: According to Livingstone and Hubel (1987), surface properties, such as color, and boundary properties, such as spatial frequency and orientation, are represented somewhat separately in the "blob" and "interblob" regions of striate cortex, a distinction carried forward to extrastriate region V2. Since previous studies (Duncan, 1993a, 1993b) had concerned boundary attributes, an extension to concurrent boundary and surface discriminations seemed important. 
As we shall see, the results rule out independence of boundary and surface systems. In most cases, results mirror those reported previously: When discriminations concern different objects, it is immaterial whether they concern the same or different attributes. Color discriminations, however, provide a partial exception, suffering reduced interference from concurrent boundary discriminations.

\section{EXPERIMENT 1}

In Experiment 1, stimuli were single, briefly presented lines varying in color (surface) and length (boundary). Two lines were presented in each display; when concurrent discriminations were made, they always concerned different lines (different objects). For one group of subjects, concurrent discriminations concerned the same attribute of the two lines (color for some subjects, length for the remainder). For a second group, concurrent discriminations concerned different attributes. To minimize response factors and measure only the accuracy of stimulus identification, responses were unspeeded two-alternative forced choices made separately for each line. Interference between concurrent discriminations was assessed by comparison with control conditions in which only a single discrimination (concerning a single line) was made.

\section{Method}

Subjects. Twelve subjects ( 8 females, 4 males; between 21 and 42 years old) were recruited from the Applied Psychology Unit's panel of paid volunteers. Each subject served in a single session of about $1 \mathrm{~h}$.

Apparatus. The subjects were tested singly in a semidarkened room. Displays were presented on a color monitor (Digivision CD 14) under control of a Cambridge Electronic Design laboratory computer interfaced to a Pluto Megares 1 graphics controller. Displays were always bright on a black background. Custom software allowed control of stimulus exposures to the nearest $20 \mathrm{msec}$ (frame time). Viewing distance, fixed by a chinrest, was approximately $85 \mathrm{~cm}$. Responses were made on a purpose-built fourfinger keyboard interfaced to the computer, using middle and index fingers of each hand; a footswitch, similarly interfaced, was used to initiate each trial.

Task and Procedure. Each trial began with a central fixation point accompanied by four "frames" indicating possible stimulus positions. Each frame was a $1^{\circ} 12^{\prime} \times 1^{\circ} 0^{\prime}$ box of four dots (Figure 1 ), centered $1^{\circ} 32^{\prime}$ from fixation; frame positions (left, right, top, bottom) are shown in Figure 1. All 17 dots in this display were white.

When adequately fixated, the subject pressed the footswitch to present the stimulus display. The fixation display blinked off for $60 \mathrm{msec}$, then reappeared along with the experimental stimuli (Figure 1, top). These stimuli were two lines-one (the horizontal stimulus) presented unpredictably in the left or right frame, the other (the vertical stimulus) unpredictably in the top or bottom frame. Unpredictable positions were used for each stimulus simply to ensure central fixation; they were always irrelevant to the task. The horizontal stimulus was either red $(x=.630, y=.332$, $Y=9.2$ according to the CIE system; measurements made on large patches with a Minolta TV Color Analyzer II) or orange $(x=.517, y=.420, Y=11.8)$ and was either $0^{\circ} 49^{\prime}$ or $0^{\circ} 26^{\prime}$ in length. It was centered $0^{\circ} 9^{\prime}$ above the center of its frame and rotated $4^{\circ} 46^{\prime}$ clockwise from the horizontal. (Position and orientation were set for comparability with other experiments in which these attributes varied; they were not relevant here.) The vertical
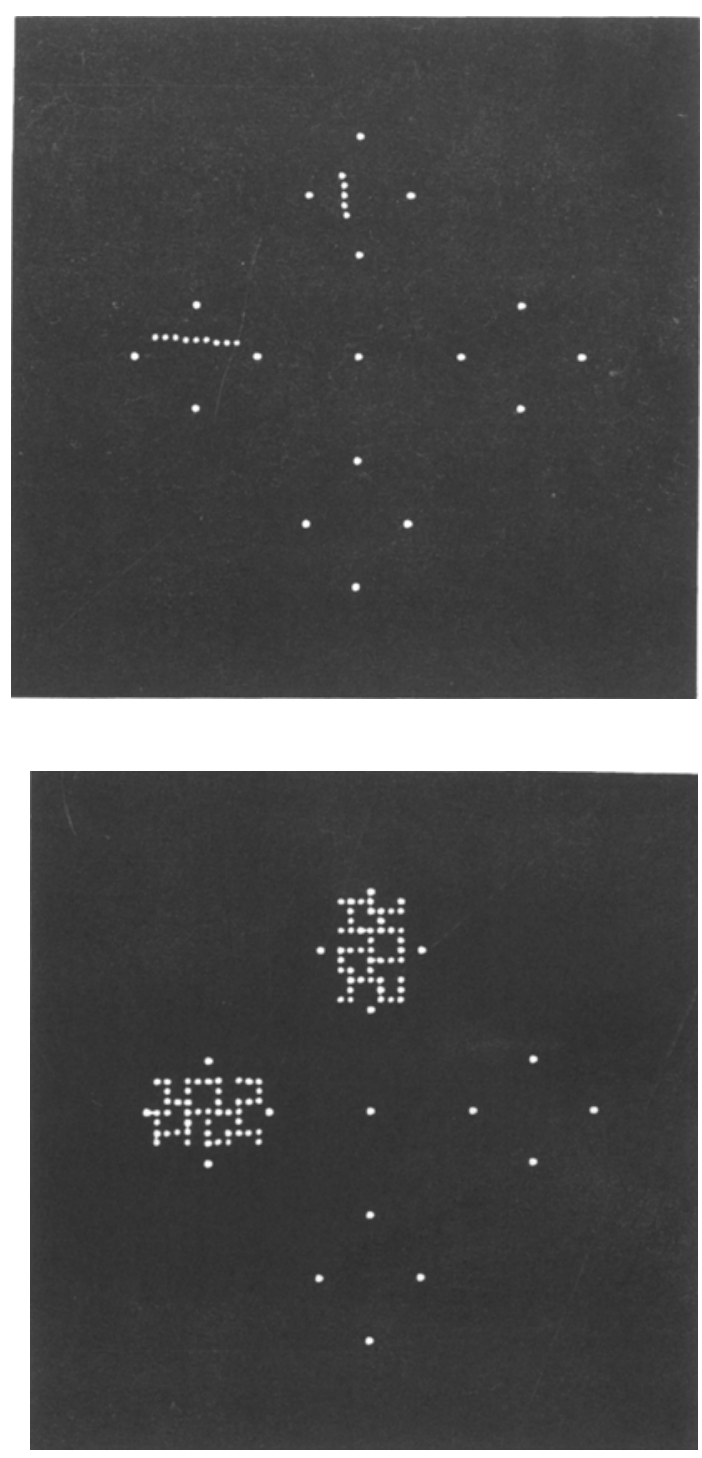

Figure 1. Example stimulus (top) and mask (bottom) displays from Experiment 1.

stimulus was either green $(x=.297, y=.555, Y=27.3)$ or blue $(x=.235, y=.353, Y=28.8)$ and again $0^{\circ} 49^{\prime}$ or $0^{\circ} 26^{\prime}$ in length. It was centered $0^{\circ} 9^{\prime}$ to left of center within its frame and rotated $4^{\circ} 46^{\prime}$ counterclockwise from the vertical.

After a predetermined exposure duration, stimulus lines were immediately replaced by masks centered within the corresponding frames (Figure 1, bottom). Each mask was a $1^{\circ} 0^{\prime} \times 0^{\circ} 37^{\prime}$ patch of jumbled contour, drawn with a mixture of red, orange, green, and blue pixels (colors as above). Masks, still accompanied by the fixation display, remained for $500 \mathrm{msec}$, after which the screen went blank until responses were complete. A further blank interval of $1 \mathrm{sec}$ preceded onset of fixation for the next trial.

For each subject, only one attribute of each stimulus varied across trials, and only this attribute was ever relevant. For 3 subjects, the relevant attribute for both lines was color. For 3 subjects, the relevant attribute for both lines was length. For 3 subjects, relevant attributes were color for the horizontal line and length for the vertical line. For 3 subjects, this arrangement was reversed. Altogether then, 6 subjects (same-attribute group) had the same relevant attribute for both stimuli, and 6 subjects (different-attribute 
group) had different relevant attributes. When not varying, colors were fixed at red (horizontal stimulus) and green (vertical stimulus), and lengths at $0^{\circ} 49^{\prime}$.

Each subject served in three conditions, presented in separate blocks of trials. In the divided attention, or dual-discrimination, condition, the relevant attributes of both stimuli were to be identified on each trial. Responses for the horizontal line were made first using the pair of keys operated by the left hand-the left key within this pair indicating red or long, the right key indicating orange or short. Responses for the vertical line were made second using the pair of keys operated by the right hand-the left key within this pair indicating green or long, the right key indicating blue or short. In the remaining two single-discrimination conditions, only one attribute was identified on each trial -in one case, just the relevant attribute of the horizontal line (left hand response), and in the other case, just the relevant attribute of the vertical line (right hand response). It is worth emphasizing that, in this experiment, the number of discriminations was perfectly confounded with the number of attended objects; single- and dual-discrimination conditions required attention to one and two objects, respectively. In all conditions, accuracy rather than speed was emphasized, and the subjects were strongly discouraged from responding so quickly as to make keyboard errors. Since similar methods have shown equal accuracy in single- and dual-discrimination conditions, providing that both discriminations concern the same object (Duncan, 1984, 1993a), simple keyboard errors can be discounted as a source of dual-discrimination performance decrement.

The session began with a block of practice in each condition, the order of conditions being counterbalanced within each group of 3 subjects. Each block was divided into three subblocks-the first two having 12 trials each, the third having 24. Exposure durations in the three subblocks were 240,100 , and $60 \mathrm{msec}$, respectively (stimulus onset asynchronies between lines and masks). After practice, a fixed exposure duration was chosen for the remainder of the experiment, aiming for $80 \%-95 \%$ correct in singlediscrimination conditions. Exposure durations ranged from 80 to $140 \mathrm{msec}$; means for same- and different-attribute groups were 103 and $110 \mathrm{msec}$, respectively. Data were then collected in one further block of trials per condition, with the same order of conditions as before. Each block was divided into two subblocks, with 56 and 48 trials respectively; the first 8 trials of the first sub-block were discarded. At the end of each practice or experimental subblock, percentage correct for that subblock was shown on the screen for $10 \mathrm{sec}$. For each subblock, a new sequence of trials was constructed. Sequences were random except that, within the last 48 trials of each experimental subblock, each of the 16 possible combinations of relevant attribute values and stimulus locations (left or right for horizontal, top or bottom for vertical) appeared three times.

\section{Results}

The mean proportion correct in each condition is shown in Table 1. Performance decrements due to divided attention (difference between single- and dual-discrimination conditions) are shown in the third column. For example, a subject identifying the color of both lines would contribute both horizontal and vertical line data to the top section (same attribute, color) of the table, and a subject identifying color of the horizontal line and length of the vertical line would contribute horizontal line data to the third section (different attribute, color) and vertical line data to the fourth section (different attribute, length).

The data show a surprising result. For color discriminations, only the same-attribute group showed a dualdiscrimination decrement. These are the results we should
Table 1

Proportion Correct in Experiment 1

Condition

Single Dual

Discrimination Discrimination Decrement

Same Attribute

Color

$\begin{array}{llll}\text { Horizontal } & .955 & .872 & .083 \\ \text { Vertical } & .955 & .816 & .139 \\ \text { Mean } & .955 & .844 & .111 \\ \text { Length } & & & \\ \text { Horizontal } & .896 & .854 & .042 \\ \text { Vertical } & .840 & .597 & .243 \\ \text { Mean } & .868 & .726 & .142 \\ & \text { Different Attribute }\end{array}$

Color

$\begin{array}{llrr}\text { Horizontal } & .986 & .955 & .031\end{array}$

$\begin{array}{llll}\text { Vertical } & .816 & .955 & -.031 \\ \text { Mean } & .901 & .851 & -.035\end{array}$

$\begin{array}{llll}\text { Mean } & .901 & .903 & -.002\end{array}$

Length

$\begin{array}{llll}\text { Horizontal } & .813 & .719 & .094\end{array}$

$\begin{array}{llll}\text { Vertical } & .882 & .663 & .219\end{array}$

Mean

.848

.691

.157

expect if surface and boundary systems were functionally independent. For size discriminations, in contrast, same- and different-attribute groups showed the same dual-discrimination decrement. These results match those previously obtained for boundary attributes: Decrements are unaffected by the similarity of concurrent discriminations (Duncan, 1993a, 1993b).

Separate analysis of color and length data is not straightforward, since horizontal/vertical is a within-subjects factor for half of the subjects (same-attribute group) but a between-subjects factor for the others (different-attribute group). To address the problem, $t$ statistics were calculated using appropriate composite error terms whose approximate degrees of freedom were given by the formulas of Satterthwaite (1946). These degrees of freedom are nonintegral, depending not only on the degrees of freedom of the component error terms but also on their relative sizes. Color and length data were examined by separate sets of these $t$ tests, in each case assessing the main effect of divided attention (single vs. dual discriminations) and its interactions with similarity (same vs. different attributes) and stimulus (horizontal vs. vertical).

For the color data, the main effect of attention $[t(5.04)=$ $2.15, p<.09]$ and its interaction with similarity $[t(5.04)=$ $2.22, p<.08]$ were both marginally significant. The interaction of attention with stimulus was not $[t(5.48)=$ $0.14]$. For the length data, the main effect of attention was significant $[t(4.78)=9.84, p<.001]$, along with its interaction with stimulus $[t(3.15)=3.84, p<.05]$. The interaction with similarity was not $[t(4.78)=0.46]$.

\section{Discussion}

Two contrasting predictions were available for this experiment. According to the simple object-based model, dual-discrimination decrements should have been equal in same- and different-attribute groups (Duncan, 1993a, 
1993b). According to this model, the key consideration is the number of relevant objects, not the nature of discriminations that are made. In contrast, the possibility of parallel, independent function in surface and boundary systems predicted that dual-discrimination decrements would be reduced or eliminated in the different-attribute case. In the event, neither prediction was upheld. For the boundary discrimination (length), the predictions of the object-based model were upheld. For the surface discrimination (color), results matched the predictions of parallel, independent systems.

To back up these conclusions-in particular, those based on marginal significance-three replications of the basic results are summarized in Table 2. In each case, the design was much as that for Experiment 1; only mean dual-discrimination decrements are shown. In the first experiment (Tach 1 ), stimuli were presented in a threefield tachistoscope, and responses were made orally. Stimuli were a box and a line, overlapping and both centered on fixation. They were drawn in colored ink on a white background. The box measured $0^{\circ} 14^{\prime}$ in width and $1^{\circ} 16^{\prime}$ or $1^{\circ} 36^{\prime}$ in height; it was red or orange. The line, horizontally aligned, measured $0^{\circ} 38^{\prime}$ or $0^{\circ} 48^{\prime}$ in length; it was green or blue. Stimulus exposure was $50 \mathrm{msec}$, followed immediately by a mask of jumbled contour. There were 12 subjects in the same-attribute group ( 6 identifying both colors, 6 both lengths), 12 in the differentattribute group ( 6 identifying box color and line length, 6 the reverse), and 12 more who identified the two attributes of a single object ( 6 box, 6 line). For each subject, there were 96 trials per condition (one dual-discrimination, two single-discrimination conditions), following 144 trials per condition of practice. The second experiment (Tach2) was closely similar, except that the overlapping stimuli were an elongated Necker cube, aligned horizontally, and the letter $\mathrm{H}$, aligned vertically. The elongated cube measured $0^{\circ} 45^{\prime}$ vertically and $2^{\circ} 0^{\prime}$ or $2^{\circ} 28^{\prime}$ horizontally; it was red or orange. The $\mathrm{H}$ measured $0^{\circ} 38^{\prime}$ horizontally and $1^{\circ} 16^{\prime}$ or $1^{\circ} 36^{\prime}$ vertically; it was green or blue. Stimulus exposure was $70 \mathrm{msec}$, with masking as before. There were 72 trials per condition, following 52 trials per condition of practice. Groups and number of subjects per group were as in Tachl. The third experi-

Table 2

Dual-Discrimination Decrements in Three Replications of Experiment 1

\begin{tabular}{llcc}
\hline & \multicolumn{3}{c}{ Experiment } \\
\cline { 2 - 3 } & \multicolumn{1}{c}{ Tach1 } & Tach2 & Move \\
\hline Color & \multicolumn{2}{c}{ Same Attribute } & \\
Length & .092 & .112 & .071 \\
& .064 & .116 & .091 \\
Color & Different Attribute & \\
Length & .007 & .031 & .017 \\
& .019 & .101 & .123 \\
Color & \multicolumn{2}{c}{ Single Object } & \\
Length & -.031 & .004 & \\
\hline
\end{tabular}

ment (Move) used the same apparatus as that in Experiment 1. Stimuli were an overlapping box and line similar to those of Tach1, with similar colors and lengths. For half of the subjects, an attempt was made to break up perceptual grouping by having the line move continuously upward across the box during the stimulus exposure ( $80 \mathrm{msec}$, masked as before); in the event, this had little effect on the results. There were 12 subjects each in same- and different-attribute groups, with no subjects this time identifying both attributes of one object. For each subject, there were 96 trials per condition, following 56 trials per condition of practice. Taking all experiments together, there is little doubt that the basic result is robust. For length, the similarity of concurrent discriminations does not matter. Dividing attention between objects produces the same cost whether the two discriminations required are the same or different. For color, similarity is crucial. An analysis of variance (ANOVA) on color data from same-attribute groups was based on 6 subjects per experiment, each subject providing data for both objects. There was a highly significant cost of divided attention $[F(1,15)=45.4, p<.001]$. An ANOVA on color data from different-attribute groups was based on 12 subjects per experiment, each subject providing data for just one object or the other. The cost of divided attention was not significant $[F(1,30)=2.2, p>.10]$. As in previous studies, finally, all divided attention costs disappeared when concurrent discriminations concerned the same object (Tach1 and Tach2).

One possibility is that, in the different-attribute case, the subjects paid attention or allocated processing capacity preferentially to the object whose color was to be identified. This might have eliminated dual-discrimination decrements for that object, but it should have increased them for the other object (the length discrimination). The data in Tables 1 and 2 do not support this possibility; for length discriminations, decrements were no greater in the different-attribute case than in the same-attribute case. In more detail, the data suggest that the subjects always favored whichever object they were to report on first (Duncan, 1984, 1993a, 1993b) and that this tendency was much the same whether they made two length discriminations or one length and one color. Consider again the dual-discrimination decrements for length discriminations in Experiment 1. On the horizontal line (reported first), decrements were .042 and .094 , respectively, in same- and different-attribute groups. On the vertical line (reported second), decrements were .243 and .219. Averaging over the other three experiments, length discrimination decrements for the first-reported object were .025 (same attribute) and .050 (different attribute); for the second-reported object, they were .155 and .112 . These findings suggest that, even in the different-attribute case, processing capacity was allocated preferentially to the object reported first. Size discriminations were affected by this preferential allocation; color discriminations, however, were not. For these discriminations, there was no interference even when they were reported second 
(i.e., when attention was directed preferentially to a different object).

\section{EXPERIMENT 2}

A possibility arising from Experiment 1 is asymmetrical interference between surface and boundary discriminations. Making either a surface or a boundary discrimination on one object interferes with boundary discriminations on another. Surface discriminations, in contrast, suffer interference only from other surface discriminations.

To test this hypothesis, new surface attributes were introduced in Experiment 2. Experiment $2 \mathrm{~A}$ dealt with brightness, color, and length. In addition to same-attribute groups, we examined concurrent discriminations of brightness and color and of brightness and length. The hypothesis predicts substantial dual-discrimination decrements in the first case (two surface discriminations) but a reduced decrement for brightness in the second case. Experiment $2 \mathrm{~B}$ had the same design, but this time substituting texture (a new surface discrimination) for brightness and substituting location (a new boundary discrimination $^{1}$ ) for length.

\section{Method}

Subjects. Forty-eight subjects ( 36 females, 12 males; between 18 and 46 years old) were recruited as in Experiment 1 . Twenty-four subjects served in Experiment 2A, and 24 served in Experiment 2B.

Apparatus. The same apparatus and conditions were used as in Experiment 1.

Tasks and Procedure. In Experiment $2 \mathrm{~A}$, stimuli were closely similar to those of Experiment 1. The 24 subjects were divided into two groups of 12: the surface-surface (S-S) and the surfaceboundary $(\mathrm{S}-\mathrm{B})$ groups. For each group of 12 subjects, the design matched that of Experiment 1.

In the S-S group, relevant attributes were brightness and color. When the horizontal line varied in brightness, it was either $\operatorname{dim}(x=$ $.628, y=.336, Y=3.8)$ or bright $(x=.630, y=.332, Y=9.2)$ red. When this line varied in color, the same red and orange were used as in Experiment 1. When the vertical line varied in brightness, it was either $\operatorname{dim}(x=.304, y=.585, Y=37.6)$ or bright $(x=.299$, $y=.586, Y=98.8)$ green. Very different brightnesses were chosen for horizontal and vertical lines to discourage relative judgments between them. For variation in vertical line color, colors again were copied from Experiment 1. Note again that, for any given subject and line, either brightness varied and was relevant while color was fixed or vice versa. For 3 subjects, the relevant attribute of both lines was brightness; for 3 other subjects, it was color. For 3 subjects, relevant attributes were horizontal brightness and vertical color; for 3 other subjects, they were horizontal color and vertical brightness. For all lines, length was fixed at $0^{\circ} 49^{\prime}$.

In the S-B group, relevant attributes were brightness and length. The horizontal line was always red, and the vertical line was always green. When brightness varied, the values described above were employed and lines measured $0^{\circ} 49^{\prime}$. When length varied, stimuli matched those of Experiment 1 in both (variable) length and (fixed) color and brightness. Again, the subjects were divided into groups identifying both brightnesses, both lengths, horizontal brightness and vertical length, or horizontal length and vertical brightness.

In Experiment 2B, this whole design was repeated, substituting texture for brightness and location for length. This time, stimuli were not lines but were patches of checkerboard, measuring

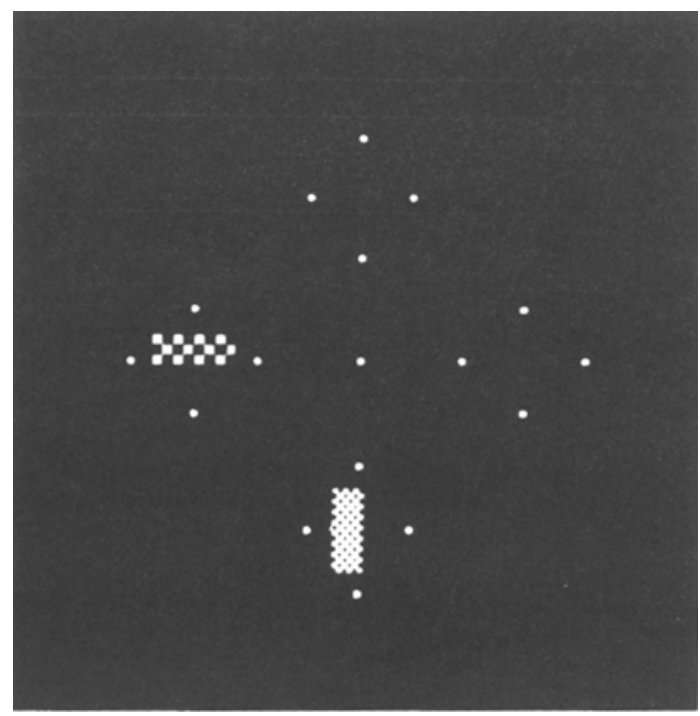

Figure 2. Example stimulus display from Experiment 2B.

$0^{\circ} 46^{\prime} \times 0^{\circ} 17^{\prime}$ (Figure 2). The texture of the checkerboard was fine (10.4 cycles/deg) or coarse $(5.2 \mathrm{cycles} / \mathrm{deg})$. Colors for the horizontal patch were red $(x=.634, y=.328, Y=8.8)$ or orange $(x=.576, y=.372, Y=7.9)$; colors for the vertical patch were green $(x=.256, y=.419, Y=17.3)$ or blue $(x=.219, y=.297$, $Y=18.2$ ). These were also the colors used to construct the backward mask. Within its frame, the horizontal patch was located $0^{\circ} 6^{\prime}$ either above or below center; the vertical patch was located a similar distance to left or right of center. As before, each patch varied in only one attribute for any given subject; when not varying, texture was fine, colors were red and green, and locations were above and left. In the $\mathrm{S}-\mathrm{S}$ group, 3 subjects identified the texture of both patches, 3 identified the color of both patches, 3 identified horizontal texture and vertical color, and 3 identified horizontal color and vertical texture. In the S-B group, 3 subjects identified the texture of both patches, 3 identified the location of both patches, 3 identified horizontal texture and vertical location, and 3 identified horizontal location and vertical texture.

Other details followed Experiment 1. Responses for the horizontal stimulus were made with the left hand; within the pair of keys, the left one was used to indicate dim, red, or short in Experiment $2 \mathrm{~A}$, and fine, red, or above in Experiment $2 \mathrm{~B}$. Responses for the vertical stimulus were made with the right hand; within the pair of keys, the left one was used to indicate dim, green, or short in Experiment 2A, and fine, green, or left in Experiment 2B. Exposure durations in experimental blocks ranged from 60 to $140 \mathrm{msec}$, with means of 88 and $91 \mathrm{msec}$, respectively, for sameand different-attribute subjects. In Experiment 2B, the blink in the fixation display preceding stimulus onset was increased from 60 to $160 \mathrm{msec}$ to accommodate the increased construction time of the larger stimuli.

\section{Results}

Results for the $\mathrm{S}-\mathrm{S}$ groups in both Experiments $2 \mathrm{~A}$ and 2B are shown in Table 3 . To simplify the table, only average results for horizontal (first-reported) and vertical (second-reported) stimuli are shown. The results were largely as expected, given that all discriminations concerned surface attributes. In all cases, there was a dualdiscrimination decrement, much the same in magnitude whether identified attributes were the same or different. 
Table 3

Proportion Correct in S-S Groups in Experiment 2

\begin{tabular}{lccc}
\hline & \multicolumn{2}{c}{ Condition } & \\
\cline { 2 - 3 } & $\begin{array}{c}\text { Single } \\
\text { Discrimination }\end{array}$ & $\begin{array}{c}\text { Dual } \\
\text { Discrimination }\end{array}$ & Decrement \\
\hline \multicolumn{3}{c}{ Same Attribute } & \\
Color & & & \\
$\quad$ Experiment 2A & .868 & .813 & .055 \\
Experiment 2B & .875 & .809 & .066 \\
Mean & .872 & .811 & .061 \\
Other & & & \\
Brightness (2A) & .867 & .752 & .115 \\
Texture (2B) & .874 & .769 & .105 \\
Mean & .871 & .761 & .110 \\
& Different Attribute & \\
Color & & & \\
Experiment 2A & .768 & .712 & .056 \\
Experiment 2B & .952 & .875 & .077 \\
Mean & .860 & .794 & .066 \\
Other & & & \\
Brightness (2A) & .686 & .623 & .063 \\
Texture (2B) & .906 & .750 & .156 \\
Mean & .796 & .687 & .109 \\
\hline
\end{tabular}

Color data were examined by a series of $t$ tests, using the same methods as before. Of most importance, there was a significant main effect of divided attention $[t(9.6)=$ $2.40, p<.05]$, but no interaction with similarity (same vs. different attributes) $[t(9.6)=0.10]$ or with stimulus (horizontal vs. vertical) $[t(8.3)=0.06]$. Interactions between each of these effects and experiment (Experiment $2 \mathrm{~A}$ vs. Experiment $2 \mathrm{~B}$ ) were also nonsignificant (all $t \mathrm{~s} \leq 0.53$ ). Combined brightness and texture data were analyzed similarly. Results were much the same, with a significant main effect of divided attention $[t(11.1)=3.02, p<.02]$, but no interaction with either similarity $[t(11.1)=0]$ or stimulus $[t(8.8)=1.53]$ and no higher order interactions with experiment (all $t \mathrm{~s} \leq 0.71$ ).

Though the data from S-S groups fit the hypothesis of asymmetrical interference between surface and boundary discriminations, data from $\mathrm{S}-\mathrm{B}$ groups did not. The results are shown in Table 4. This time, the dual-discrimination decrement for surface attributes was not reduced by pairing with a boundary discrimination. Instead, decrements in same- and different-attribute groups were similar, just as they were in the S-S data. Analyses conducted as before confirmed these results. For combined surface attribute data (brightness and texture), there was a significant main effect of divided attention $[t(7.2)=4.55, p<$ $.005]$, but no interaction with similarity $[t(7.2)=0.27]$ or stimulus $[t(7.4)=1.54]$ and no higher order interactions with experiment (all $t \mathrm{~s} \leq 0.96$ ). For combined boundary attribute data (length and location), there was a significant main effect of divided attention $[t(12.0)=7.12, p<$ $.001]$, but no interaction with similarity $[t(12.0)=0.61]$ or stimulus $[t(8.7)=1.52]$. This time, there was a significant interaction between divided attention and experiment $[t(12.0)=2.85, p<.02]$, reflecting greater overall decrements in Experiment 2B (i.e., for discriminations of location as compared with length). Especially large decrements for location discriminations have been noted before (Duncan, 1993a). Higher order interactions with experiment were not significant (both $t \mathrm{~s} \leq 0.36$ ).

\section{Discussion}

The hypothesis of a general asymmetry between surface and boundary discriminations is ruled out by these data. For brightness and texture, dual-discrimination decrements were not reduced by pairing with a boundary discrimination.

Two other hypotheses are also ruled out. One possibility might have been that color is only sensitive to interference from a second, concurrent color discrimination. This is ruled out by interference from other, concurrent surface discriminations-brightness and texture-in S-S groups (Table 3). An ANOVA restricted just to the color data of $\mathrm{S}-\mathrm{S}$ different-attribute subjects showed significant interference from concurrent discriminations of brightness and texture $[F(1,8)=14.6, p<.01]$. A second possibility might have been that length in general does not interfere with other discriminations. Relevant data come from the S-B different-attribute subjects of $\mathrm{Ex}=$ periment $2 \mathrm{~A}$, who made concurrent discriminations of length and brightness (Table 4). An ANOVA restricted just to the brightness data of these subjects showed significant interference from a concurrent length discrimination $[F(1,4)=35.2, p<.01]$. Length has also been shown to produce normal interference with concurrent discriminations of location, shape, orientation, and spatial frequency (Duncan, 1993a, 1993b).

It is also reassuring that results were very similar in Experiments $2 \mathrm{~A}$ and $2 \mathrm{~B}$. For example, (intended) changes in brightness may possibly introduce small (unintended) changes in color (and vice versa), a potential complicating factor in Experiment 2A. Replication of results with

\section{Table 4}

Proportion Correct in S-B Groups in Experiment 2

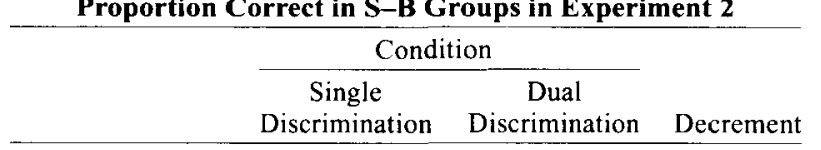

\begin{tabular}{llll} 
& Same Attribute & \\
Surface & & \\
Brightness (2A) & .832 & .728 & .104 \\
Texture (2B) & .905 & .771 & .134 \\
$\quad$ Mean & .869 & .750 & .119 \\
Boundary & & & \\
$\quad$ Length (2A) & .872 & .759 & .113 \\
Location (2B) & .908 & .648 & .260 \\
Mean & .890 & .704 & .186 \\
& Different Attribute & \\
Surface & & & \\
Brightness (2A) & .870 & .776 & .094 \\
Texture (2B) & .906 & .789 & .117 \\
$\quad$ Mean & .888 & .783 & .105 \\
Boundary & & & \\
$\quad$ Length (2A) & .851 & .719 & .132 \\
$\quad$ Location (2B) & .927 & .616 & .311 \\
$\quad$ Mean & .889 & .668 & .221 \\
\hline
\end{tabular}


different perceptual attributes minimizes their possible dependence on unwanted cues.

In fact, the results of Experiment 2 were precisely in accord with previous findings on boundary discriminations (Duncan, 1993a, 1993b) and with the predictions of the object-based model. Identifying attributes of two objects always produced exactly the same interference, whether these attributes were the same or different.

Though not shown in Tables 3 and 4, it was true that dual-discrimination decrements were greater overall for the vertical stimulus (reported second; mean decrement $=.151$ ) than for the horizontal stimulus (reported first; mean decrement $=.094)$. A trend in this direction $(t \geq$ 1.52) was apparent in three of the four sets of $t$ tests described above. Again, these data suggest that, in dualdiscrimination conditions, processing capacity was allocated preferentially to the object reported first.

\section{EXPERIMENT 3}

So far, the results tend to point to some specific peculiarity of color discriminations. Under certain circumstances, these discriminations escape the normal effects of competition between objects for attention. There is no dual-discrimination deficit, and there is no effect of attentional bias toward one object or the other. Competition is effective only when color discriminations are paired with other, surface discriminations on a second object.

As outlined above, a part of the motivation for distinguishing surface and boundary processes is neurophysiological. Even stronger, perhaps, is the separation of color and motion. From the retina, through the lateral geniculate to the cortex, there is separation of a "magnocellular" pathway, characterized by blindness to absolute color but high motion sensitivity, and a "parvocellular" pathway, characterized by color vision but lower motion sensitivity. Lesions of the magnocellular pathway have no effect on color discrimination but a substantial effect on motion discrimination; lesions of the parvocellular pathway leave motion discrimination unaffected but reduce color discrimination to chance (Schiller \& Logothetis, 1990). To examine further the peculiarities of color, color and motion discriminations were paired in Experiment 3.

\section{Method}

Subjects. Twenty-four subjects ( 19 females, 5 males; between 21 and 58 years old) were recruited as before.

Apparatus. Apparatus and conditions were as in Experiment 1 .

Tasks and Procedure. Again, each display contained two stimuli-one randomly to left or right of fixation (horizontal stimulus), the other randomly above or below (vertical). This time, each stimulus was a patch of $22 \mathrm{dots}$, spread approximately evenly within a $1^{\circ} 29^{\prime}$ square area centered $3^{\circ} 36^{\prime}$ from fixation (Figure 3 ). Dot size was $0^{\circ} 6^{\prime}$ horizontally $\times 0^{\circ} 3^{\prime}$ vertically. With the above constraints, a new random arrangement of dots was created for each trial.

Each stimulus varied in color and motion of the dots within it. For the horizontal stimulus, colors were red $(x=.634, y=.325$, $Y=15.4)$ or pink $(x=.510, y=.255, Y=16.0)$. Dots scrolled

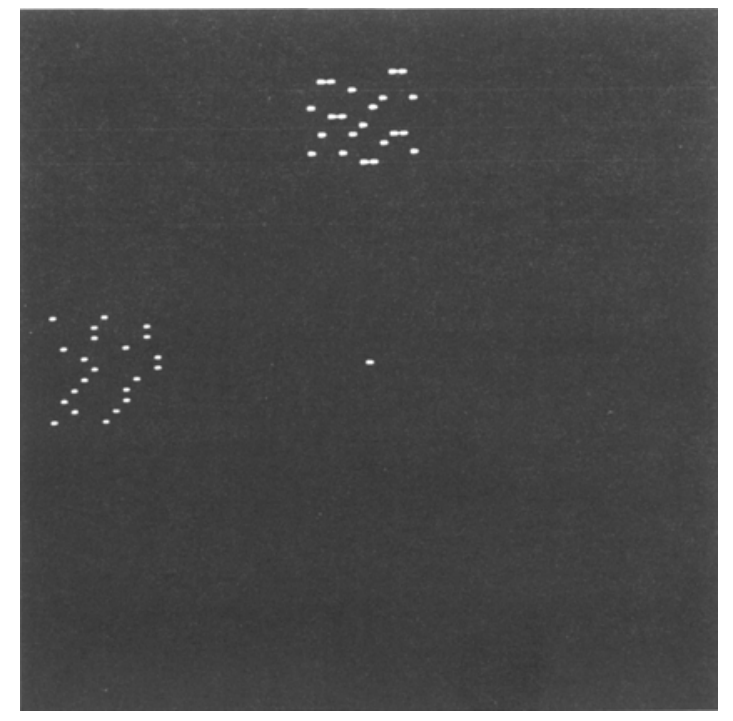

Figure 3. Example stimulus frame from Experiment 3.

left or right at a speed of $7.2 \% \mathrm{sec}$, with wraparound at the edge of the $1^{\circ} 29^{\prime}$ display window. For the vertical stimulus, colors were green $(x=.299, y=.554, Y=36.5)$ or blue $(x=.243, y=.377$, $Y=37.9$ ); dots scrolled up or down. This time, flicker photometry was used to check that, for each subject, red-pink and greenblue color pairs were close in subjective brightness (Boynton, 1979). Stimuli like those from the experiment were flickered between red and pink or between green and blue at rates from 25.0 to $6.3 \mathrm{~Hz}$. The subjects judged whether displays looked flickering or steady. Most commonly, all displays were judged to be steady; only 2 subjects reported flicker at $12.5 \mathrm{~Hz}$, and none at a higher rate. Such results suggest little stimulation of the luminance channel.

The design was as before, with only one attribute of each stimulus varying for each subject. Six subjects identified the color of both stimuli, 6 identified the motion of both, 6 identified horizontal color and vertical motion, and 6 identified horizontal motion and vertical color. This time, 3 subjects in each group made the horizontal response first in the dual-discrimination condition, and 3 made the vertical response first. Again, the first response was always made with the left-hand pair of keys. When fixed, colors were set to red and green and motions were set to left and up. These were also the values used for the left response within each key pair.

Practice and experimental blocks were similar to those of Experiments 1 and 2 . In this experiment, the fixation display consisted only of a central dot, without frames to indicate possible stimulus positions, and blinked off for only $40 \mathrm{msec}$ before onset of the stimulus display. In the practice blocks, stimulus exposure duration was always $80 \mathrm{msec}$ ( 4 frames), with an interstimulus interval (ISI) of 160,80 , or $0 \mathrm{msec}(8,4$, or 0 frames) preceding mask onset in the three successive subblocks of each condition. In the experimental blocks, exposure duration ranged from 60 to $80 \mathrm{msec}$ (mean $=68 \mathrm{msec}$ ), and ISI ranged from 0 to $40 \mathrm{msec}$ (mean $=8 \mathrm{msec}$ ), with very similar values for same- and differentattribute groups. Mask size was increased from that of Experiments 1 and 2 to $1^{\circ} 35^{\prime}$ square; mask patches were again centered on the same location as preceding stimuli. In all other respects, the procedure matched that of Experiment 1.

\section{Results}

Mean proportion correct in each condition is shown in Table 5. Results are shown separately for stimuli reported 
first and second in the dual-discrimination condition. For example, a subject reporting horizontal color followed by vertical color would contribute horizontalstimulus data to the first row of the table and verticalstimulus data to the second row. The results were very similar to those obtained with color and length discriminations in Experiment 1. For color, the dual-discrimination decrement was all but eliminated in the different-attribute case. For motion, the decrement was unaffected by similarity of discriminations.

Color data were analyzed as before. There was a significant main effect of divided attention [ $t(13.68)=$ $4.38, p<.001]$ and a significant interaction with similarity $[t(13.68)=3.03, p<.01]$, but not stimulus (first or second) $[t(14.56)=0.90]$. For motion, there was a significant main effect of divided attention $[t(14.97)=$ $3.55, p<.01]$, but no interaction with either similarity $[t(14.97)=0.20]$ or stimulus $[t(13.20)=0.58]$.

\section{Discussion}

These results confirm asymmetrical interference between color and nonsurface discriminations. Paired with motion, color again escapes the usual two-object interference. At the same time, the color discrimination produces interference on concurrent motion discrimination.

Very similar results were obtained in a second experiment using similar stimuli and procedures. This time, there were 12 single-attribute subjects, 12 differentattribute subjects, and 12 subjects identifying color and motion of a single stimulus ( 6 horizontal, 6 vertical). In the same-attribute case, dual-discrimination decrements were .090 and .125 , respectively, for color and motion. In the different-attribute case, they were .037 and .127 . In the single object case, they were -.012 and .026 .

An implication of the object-based model is coordination between physiologically distinct subsystems working

Table 5

Proportion Correct in Experiment 3

\begin{tabular}{|c|c|c|c|}
\hline & \multicolumn{2}{|c|}{ Condition } & \multirow[b]{2}{*}{ Decrement } \\
\hline & $\begin{array}{c}\text { Single } \\
\text { Discrimination }\end{array}$ & $\begin{array}{c}\text { Dual } \\
\text { Discrimination }\end{array}$ & \\
\hline \multicolumn{4}{|c|}{ Same Attribute } \\
\hline \multicolumn{4}{|l|}{ Color } \\
\hline Ist report & .962 & .882 & .080 \\
\hline 2 nd report & .941 & .839 & .102 \\
\hline Mean & .952 & .861 & .091 \\
\hline \multicolumn{4}{|l|}{ Motion } \\
\hline 1st report & .882 & .788 & .094 \\
\hline 2 nd report & .832 & .785 & .047 \\
\hline Mean & .857 & .787 & .070 \\
\hline \multicolumn{4}{|c|}{ Different Attribute } \\
\hline \multicolumn{4}{|l|}{ Color } \\
\hline 1st report & .929 & .922 & .006 \\
\hline 2 nd report & .953 & .927 & .026 \\
\hline Mean & .941 & .925 & .016 \\
\hline \multicolumn{4}{|l|}{ Motion } \\
\hline 1 st report & .868 & .835 & .033 \\
\hline 2 nd report & .938 & .821 & .117 \\
\hline Mean & .903 & .828 & .075 \\
\hline
\end{tabular}

on the different properties of a selected object. The strong interference of color on motion discriminations provides clear evidence of such coordination; though color and motion are processed by largely separate visual subsystems, a set to identify the color of one object produces strong interference on identifying the motion of another. These are the expected results if, in attention to an object, multiple subsystems converge to work on that object's different properties, and if objects attended concurrently must compete to attain this state.

\section{OTHER CASES}

Data from two other pilot experiments may be mentioned. Both experiments used the line stimuli of Experiment 1 , varying in one case in color and location $\left(0^{\circ} 9^{\prime}\right.$ above or below center for the horizontal stimulus, to left or right of center for the vertical stimulus), and varying in the other case in color and orientation $\left(4^{\circ} 46^{\prime}\right.$ clockwise or counterclockwise from horizontal alignment for the horizontal stimulus, and from vertical alignment for the vertical stimulus). Both experiments used a different-attribute design, with discrimination of color for one object and either location or orientation for the other. Each experiment had 6 subjects. In the first experiment, the dual-discrimination decrement was substantial (.177) for location, but not (.017) for color. In the second experiment, decrements were .174 for orientation and .080 for color. Though reasonably substantial, even the latter color decrement was not significant by ANOVA $(p>.20)$.

Nothing strong can be concluded from such smallscale studies, and the suggestion of a color decrement even with concurrent discrimination of orientation could be investigated further. For the moment, however, we have convincing evidence of a color decrement only with concurrent surface discriminations: brightness, texture, and color itself.

\section{GENERAL DISCUSSION}

With only one exception, the present results replicated those of Duncan (1993a, 1993b) with a range of surface and boundary discriminations. When concurrent discriminations concern different objects, the same decrement in performance is produced whether these discriminations concern the same or different attributes. Such results support a model of visual attention in which different subsystems are coordinated, making the different properties of a selected object available together for report and control of behavior.

To a large extent, the results imply coordination between surface and boundary systems and between color and motion systems. The results show that boundary and surface systems tend to work concurrently on the same object - the one defining its borders, and the other filling the space so defined with such properties as brightness and texture. The two can work together without cost, but only when they work on the same object. Forced 
to work on different objects, boundary and surface systems produce substantial mutual interference.

The exception concerns the unexplained ability of color to escape some forms of two-object interference. Specifically, color suffers little interference from concurrent discriminations of motion, size, and perhaps any nonsurface attribute. There is little dual-discrimination deficit overall and little effect of attentional bias toward one object or the other. The results rule out the hypothesis of independence between surface and boundary systems: Color does produce interference on boundary discriminations, and for brightness and texture, results are just as the object hypothesis predicts. The results also rule out independence of discriminations arising in parvocellular and magnocellular systems: Color produces substantial interference with motion. Finally, the results rule out any general insensitivity of color to concurrent discriminations: Interference is produced by concurrent discriminations of brightness, texture, and color itself.

What the data suggest instead is that objects always compete for limited processing capacity or to enter the "attended" state. At least in Experiment 1 and perhaps also in Experiment 2, competition is biased toward the object whose property is reported first. Though there is competition even with concurrent discriminations of color and boundary attributes, the color discrimination itself somehow evades interference. In this case, competition is revealed only in the accuracy of the boundary discrimination.

Though the behavior of color is unexplained, certainly it does provide an exception to the rule that interference in these simple divided attention tasks depends only on the number of relevant objects (Duncan 1984, 1993a, 1993b). Since the rule is so general, it is important that the exception should be noted.

\section{REFERENCES}

Allport, D. A. (1971). Parallel encoding within and between elementary stimulus dimensions. Perception \& Psychophysics, 10, 104108.

Boynton, R. M. (1979). Human color vision. New York: Holt, Rinehart \& Winston.

BroadBent, D. E. (1958). Perception and communication. London: Pergamon.

Bundesen, C. (1990). A theory of visual attention. Psychological Review, 97, 523-547.

Desimone, R., \& Ungerleider, L. G. (1989). Neural mechanisms of visual processing in monkeys. In F. Boller \& J. Grafman (Eds.), Handbook of neuropsychology (Vol. 2, pp. 267-299). Amsterdam: Elsevier

DUNCAN, J. (1984). Selective attention and the organization of visual information. Journal of Experimental Psychology: General, 113, 501-517.

DUNCAN, J. (1993a). Coordination of what and where in visual attention. Perception, 22, 1261-1270.

Duncan, J. (1993b). Similarity between concurrent visual discriminations: Dimensions and objects. Perception \& Psychophysics, 54, 425-430.

DunCAN, J. (1996). Cooperating brain systems in selective perception and action. In T. Inui \& J. L. McClelland (Eds.), Attention and performance XVI (pp. 549-578). Cambridge, MA: MIT Press.

GRossberg, S. (1987). Cortical dynamics of three-dimensional form, color, and brightness perception: I. Monocular theory. Perception \& Psychophysics, 41, 87-116.

Kahneman, D., \& HeniK, A. (1977). Effects of visual grouping on immediate recall and selective attention. In S. Dornic (Ed.), Attention and performance VI (pp. 307-332). Hillsdale, NJ: Erlbaum.

LAPPIN, J. S. (1967). Attention in the identification of stimuli in complex displays. Journal of Experimental Psychology, 75, 321-328.

Livingstone, M. S., \& Hubel, D. H. (1987). Psychophysical evidence for separate channels for the perception of form, color, movement, and depth. Journal of Neuroscience, 7, 3416-3468.

NeISSER, U. (1967). Cognitive psychology. New York: AppletonCentury-Crofts.

RUMELHART, D. E. (1970). A multicomponent theory of the perception of briefly exposed visual displays. Journal of Mathematical Psychology, 7, 191-218.

Satterthwaire, F. E. (1946). An approximate distribution of estimates of variance components. Biometrics Bulletin, 2, 110-114.

SCHILler, P. H., \& Logothetis, N. K. (1990). The color-opponent and broad-band channels of the primate visual system. Trends in Neurosciences, 13, 392-398.

Treisman, A. M. (1969). Strategies and models of selective attention. Psychological Review, 76, 282-299.

Treisman, A. M., \& Souther, J. (1985). Search asymmetry: A diagnostic for preattentive processing of separable features. Journal of Experimental Psychology: General, 114, 285-310.

ZEKI, S. M. (1978). Uniformity and diversity of structure and function in rhesus monkey prestriate visual cortex. Journal of Physiology, 277, 273-290.

\section{NOTE}

1. Boundary discriminations (e.g., object size, shape, orientation) are all defined by the pattern of where discontinuities occur in the visual input, whereas surface discriminations (e.g., color, brightness) do not have this dependence on spatial arrangement. For present purposes, accordingly, the position of a line with respect to its frame was also taken as a boundary property.

(Manuscript received September 26, 1995; revision accepted for publication January 21, 1996.) 